\title{
The Black Canyon Forecast Station: Experiences And Lessons Learned
}

\author{
James M. Wilson*1, 2 , Bonnie Koehler ${ }^{3}$, Kathleen Sramek ${ }^{2}$ and Treve Henwood ${ }^{2}$ \\ ${ }^{1}$ Black Canyon Forecast Station, Delta, CO, USA; '2Delta County Memorial Hospital, Delta, CO, USA; ${ }^{3}$ Delta County Health and \\ Human Services, Delta, CO, USA
}

\section{Objective}

To evaluate the sociological effect on indigenous biological event signature recognition and community resilience due to the operational activities of an infectious disease forecast station.

\section{Introduction}

The nation's first operational infectious disease forecast station, modeled after warning protocols developed in the meteorology community, was activated in 2011. The approach was originally pioneered in Haiti following the 2010 earthquake.

\section{Methods}

We assembled global event signature and forecast libraries that reflected locally diagnosed infectious disease activity and infrastructure impact in a rural community from a public health, veterinary, and human clinical medicine perspective. The deployment site is home to a variety of infectious disease including hantavirus, plague, tularemia, and West Nile in the context of high wildlife-livestockhuman interfacing. Information derived from the issuance of forecasts coupled to situational awareness was shared with the public, local officials, public health officers, veterinarians, healthcare providers, and patients through various social media methods.

\section{Results}

Provision of 30-60-90 day forecasts for routine and non-routine endemic infectious disease activity and impact facilitated better coordination of public health messaging and daily conversation with patients in the inpatient and outpatient settings. The signature of an unusual, infrastructure-disruptive outbreak of metapneumovirus and respiratory syncytial virus was recognized and communicated with enough time to activate effective clinical mitigation protocols. Cost estimates demonstrated financial benefit at a local level to anticipating surges of infectious disease activity with enough time to mitigate patient demand. Community-wide engagement with infectious disease forecasts and live event advisories included the promotion of proactive infection control and public health surveillance and response, healthcare provider recognition of non-routine infectious disease, clinical sampling and diagnostic testing protocols, clinician and patient education, and synchronization of proactive disease reporting both in the routine daily clinical setting and in times of crisis. Col- lateral benefit of consistent messaging delivered to the public by the participating entities was noted. Community awareness of the repertoire of indigenous infectious disease activity was expanded beyond the official public health notification list. Neither issuance of infectious disease forecasts nor advisories issued during crises triggered an influx of anxious well phone calls or visits to the medical system that was deemed operationally relevant.

\section{Conclusions}

Activation of a local infectious disease forecast station modeled after a local weather station promotes routine communication of a broader array of infectious disease activity than that monitored by public health; facilitates proactive, cost effective healthcare; and enabled recognition of unusual, disruptive infectious activity with enough time to enable mitigation of clinical, infrastructure, and financial impact to the community. Routine communication of comprehensive infectious disease forecast and situational awareness information promotes community adaptive fitness to a wide variety of infectious hazards. The results suggest it is possible to transform the traditional public health model of data collection and analysis to one of transparent and open data availability to support innovative reduction in morbidity and mortality.

\section{Keywords}

biosurveillance; forecast; meteorology

\section{Acknowledgments}

The authors would like to acknowledge the tremendous contributions of the staff of Ascel Bio LLC for the design and management of the Black Canyon Forecast Station; the personal contributions of Mr. Michael Smith regarding meteorological warning operations; Delta County Memorial Hospital: Betty Kahrs, Janet Moore, Jason Cleckler, Bev Carlson, Randall Koehn, Johanna Roeber, and Tom Mingen; David Van Metre of Colorado State University and Delta County Colorado State University Agriculture Extension Agent Robbie B. LaValley; and Colorado Department of Agriculture Field Veterinarian, Dan Love DVM.

*James M. Wilson

E-mail: jwilson@ascelbio.com 